Astrodynamics

https://mc03.manuscriptcentral.com/astrod

\title{
Dust in the Jupiter system outside the rings
}

\author{
Xiaodong Liu and Jürgen Schmidt \\ Astronomy Research Unit, University of Oulu, Oulu 90014, Finland \\ Xiaodong Liu, xiaodong.liu@oulu.fi
}

\begin{abstract}
Jupiter is one of the major targets for planetary exploration, and dust in the Jovian system is of great interest to researchers in the field of planetary science. In this paper, we review the five dust populations outside the ring system: grains in the region of the Galilean moons, potential dust from plumes on Europa, Jovian stream particles, particles in the outer region of the Jovian system ejected from the irregular satellites, and dust in the region of the Trojan asteroids. The physical environment for the dust dynamics is described, including the gravity, the magnetic field and the plasma environment. For each population, the dust sources are described, and the relevant perturbation forces are discussed. Observations and results from modeling are reviewed, and the distributions of the individual dust populations are shown. The understanding of the Jovian dust environment allows to assess the dust hazard to spacecraft, and to characterize the material exchange between the Jovian moons, their surface properties and distribution of non-icy constituents.
\end{abstract}

Keywords: cicumplanetary dust; interplanetary dust; stream particles; planetary rings; Europa plume; Jupiter; Galilean moons; Trojan asteroids; meteoroids; space debris

\section{Nomenclature}

\begin{tabular}{|l|l|}
\hline$R_{\mathrm{J}}$ & reference radius of Jupiter $(71492 \mathrm{~km})$ \\
\hline $\mathrm{AU}$ & astronomical unit $\left(\approx 1.5 \times 10^{8} \mathrm{~km}\right)$ \\
\hline DDS & Dust Detection System \\
\hline
\end{tabular}

\section{Introduction}

Interplanetary and circumplanetary dust particles have typical sizes ranging from nanometers up to millimeters. Dust is widely distributed in the Jovian system, where particles were first detected by the Pioneer spacecraft in the early 1970s [1,2], and the dusty ring system was first observed by the cameras onboard the Voyager spacecraft in 1979 [3,4]. Subsequently, dust particles in the Jovian system were detected in-situ by space missions, and dust rings were observed by ground- and space-based telescopes as well as cameras onboard spacecraft. So far, eight spacecraft have made measurements/observations of dust particles/rings in the Jupiter system, including Pioneer 10 and 11 [1,2,5], Voyager 1 and 2 [3,4, 6-9], Galileo [8-23], Cassini [8, 24, 25], New Horizons [26, 27], and Ulysses [28-30]. Besides, the Hubble Space Telescope [9,31] and the ground-based Keck telescope [9, 32, 33] were used to observe the Jovian ring system.

Dust particles in the Jovian system (including the Sun-Jupiter system) are roughly divided into the following six different populations: (1) particles in the Jovian ring system including the main ring, the halo ring and two gossamer 


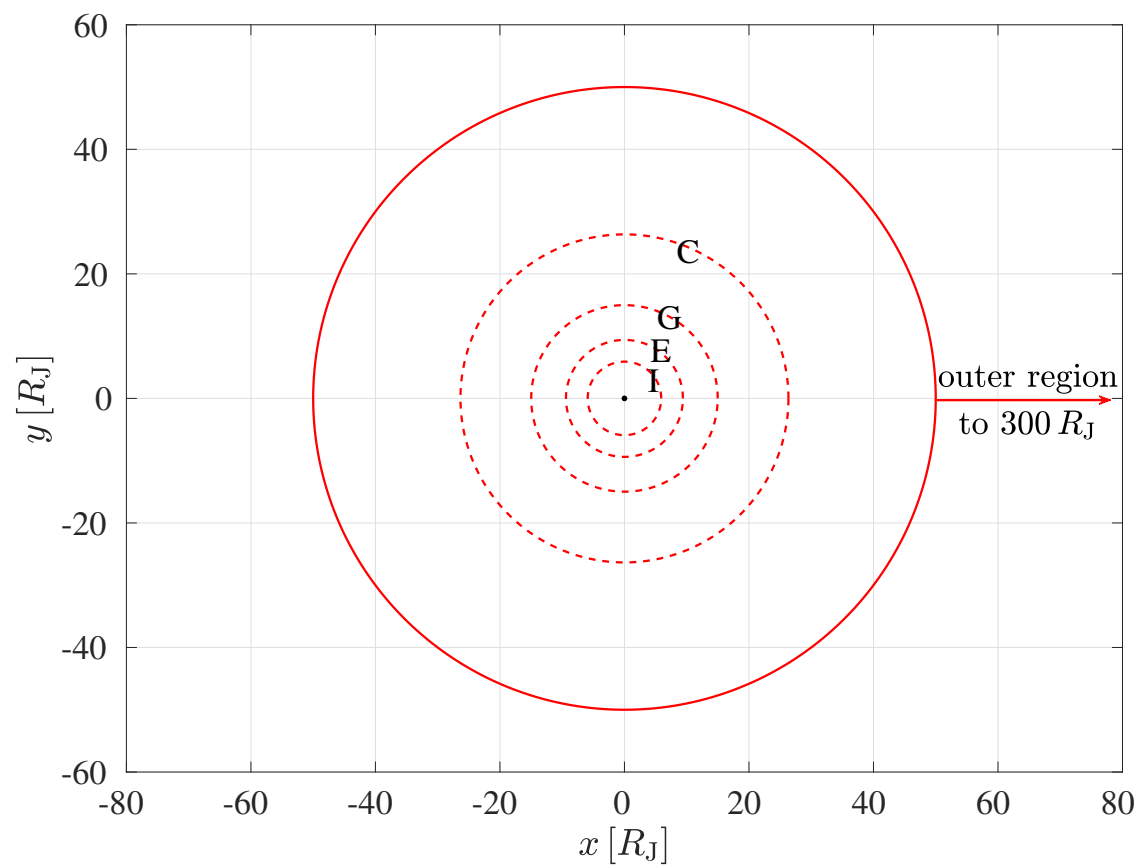

Fig. 1 Top view showing the orbits of the four Galilean moons and the outer region. Jupiter lies at the origin. The orbits of Io $\left(5.9 R_{\mathrm{J}}\right)$, Europa $\left(9.4 R_{\mathrm{J}}\right)$, Ganymede $\left(15.0 R_{\mathrm{J}}\right)$ and Callisto $\left(26.3 R_{\mathrm{J}}\right)$ are denoted as $\mathrm{I}, \mathrm{E}, \mathrm{G}$, and $\mathrm{C}$, respectively. The outer region of the Jovian system extends over a range of $[50,300] R_{\mathrm{J}}$.

rings [3, 4, 11], (2) grains in the region of the Galilean moons [21, 22], which derive from impact-generated dust clouds around the Galilean moons $[16,34]$ as well as magnetospherically captured interplanetary and interstellar particles [21, 35,36], (3) possible dust from plumes on Europa [37-41], (4) small and fast dust streams (nanometer-sized, $>200 \mathrm{~km} \mathrm{~s}^{-1}$ ) from Io's volcanic plumes [20, 29, 42], (5) particles in the outer region from the Jovian irregular moons [23], and (6) dust grains in the region of Jupiter's Trojan asteroids [43-45]. Previous studies on the first dust population, i.e. the Jovian ring system are well covered by several reviews [46-48], including a recent one published in 2018 [46], to which the reader is referred. Thus, in the current review paper we put more emphasis on the latter five populations (see Figure 1 for the locations of the four Galilean moons and the outer region of the Jovian system, and Figure 2 for the locations of the Trojan asteroids associated with the Lagrange points in the Sun-Jupiter system). The reader is also referred to an earlier review from 2004 [49], by which the dust populations (2), (4) and (5) were well covered.

In the near future, there are two reconnaissance missions that will visit the Jovian system. One is the JUpiter ICy moons Explorer mission [50] developed by European Space Agency (ESA), which is scheduled for launch in 2022. The Europa Clipper mission [51], developed by National Aeronautics and Space Administration (NASA), is planned to launch between 2022-2025. The SUrface Dust Analyzer, a dedicated dust mass spectrometer instrument, is onboard Europa Clipper. This dust detector will measure the composition of the dust particles lofted from the Galilean moons. The Lucy mission developed also by NASA will visit Jupiter's $L_{4}$ and $L_{5}$ Trojan asteroids [52], which is scheduled for launch in 2021. 


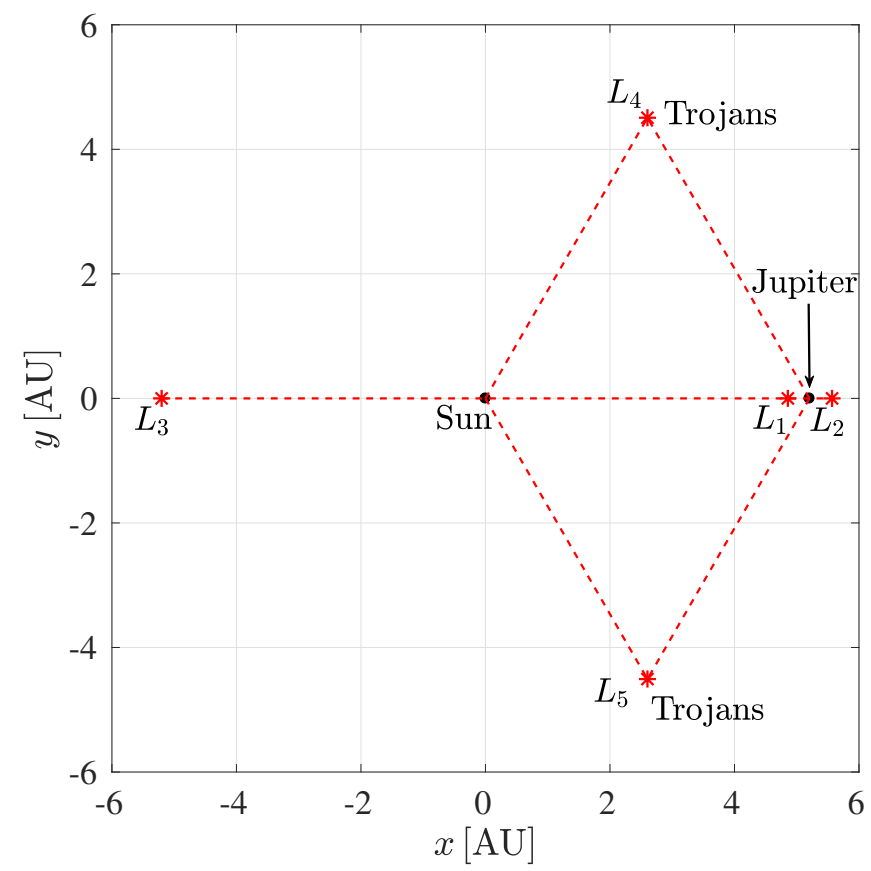

Fig. 2 Locations of the Jovian Trojan asteroids. The Sun lies at the origin. Jupiter is about 5.2 AU from the Sun. The Lagrange points in the Sun-Jupiter system are indicated as red asterisks, including three collinear Lagrange points ( $L_{1}, L_{2}$, $\left.L_{3}\right)$ and two triangular Lagrange points $\left(L_{4}, L_{5}\right)$. The $L_{4}$ and $L_{5}$ Trojan asteroids librate around the triangular Lagrange points $L_{4}$ and $L_{5}$, respectively.

\section{Physical environment for dust dynamics}

The dynamics of the dust particles in circumjovian space is influenced by various forces, including Jupiter's gravity, the Lorentz force, solar radiation pressure, Poynting-Robertson drag, plasma drag, and the gravity from other celestial bodies. Taking into account all these forces, the equation of motion of a dust grain in the Jovicentric inertial frame reads $[49,53,54]$

$$
\begin{aligned}
\ddot{\vec{r}}= & G M_{\mathrm{J}} \nabla\left\{\frac{1}{r}\left[1-\sum_{n=1}^{N_{J}} J_{2 n}\left(\frac{R_{\mathrm{J}}}{r}\right)^{2 n} P_{2 n}(\cos \theta)\right]\right\}+\frac{Q}{m_{\mathrm{g}}}\left(\dot{\vec{r}}-\vec{\Omega}_{\mathrm{J}} \times \vec{r}\right) \times \vec{B} \\
& +\frac{3 Q_{\mathrm{S}} Q_{\mathrm{pr}} \mathrm{AU}^{2}}{4\left(\vec{r}-\vec{r}_{\mathrm{S}}\right)^{2} \rho_{\mathrm{g}} r_{\mathrm{g}} c}\left\{\left[1-\frac{\left(\dot{\vec{r}}-\dot{\vec{r}}_{\mathrm{S}}\right) \cdot \hat{\vec{r}}_{\mathrm{Sd}}}{c}\right] \hat{\vec{r}}_{\mathrm{Sd}}-\frac{\dot{\vec{r}}-\dot{\vec{r}}_{\mathrm{S}}}{c}\right\} \\
& +\vec{F}_{\text {direct_drag }}+\vec{F}_{\text {Coulomb_drag }}+G m_{\odot}\left(\frac{\vec{r}_{\mathrm{dS}}}{r_{\mathrm{dS}}^{3}}-\frac{\vec{r}_{\mathrm{S}}}{r_{\mathrm{S}}^{3}}\right)+\sum_{i=1}^{N_{\mathrm{m}}} G m_{\mathrm{m}_{i}}\left(\frac{\vec{r}_{\mathrm{dm}_{i}}}{r_{\mathrm{dm}_{i}}^{3}}-\frac{\vec{r}_{\mathrm{m}_{i}}}{r_{\mathrm{m}_{i}}^{3}}\right) .
\end{aligned}
$$

Here $\vec{r}$ is the Jovicentric position vector of the particle, $G$ is the gravitational constant, $M_{\mathrm{J}}$ is the mass of Jupiter, $J_{2 n}$ is the even degree zonal of spherical harmonics for Jupiter's gravity field, $N_{J}$ is the number of $J_{2 n}, P_{2 n}$ is the Legendre polynomial of degree $2 n, \theta$ is the colatitude in the body-fixed frame, $Q$ is the grain charge, $m_{\mathrm{g}}$ is the mass of the grain, $\vec{B}$ is the local Jovian magnetic field, $\vec{\Omega}_{\mathrm{J}}$ is the angular velocity of Jupiter's rotation, $Q_{\mathrm{S}}$ is the solar radiation flux density at a heliocentric distance of one $\mathrm{AU}, Q_{\mathrm{pr}}$ is the radiation efficiency factor, $\rho_{\mathrm{g}}$ is the bulk density of the grain, $r_{\mathrm{g}}$ is the particle radius, $c$ is the light speed, $\vec{r}_{\mathrm{S}}$ is the position vector of the Sun, $\hat{\vec{r}}_{\mathrm{Sd}}$ is the unit vector along the radiation flux, $m_{\odot}$ is the mass of the Sun, $\vec{r}_{\mathrm{dS}}$ is the vector from the particle to the Sun, $N_{\mathrm{m}}$ is the number of the moons that are included as gravitational perturbers, $m_{\mathrm{m}_{i}}$ is the mass of the $i$ th moon, $\vec{r}_{\mathrm{dm}_{i}}$ 
is the vector from the particle to the $i$ th moon, and $\vec{r}_{\mathrm{m}_{i}}$ is the position vector of the $i$ th moon. The expression for the direct plasma drag $\vec{F}_{\text {direct_drag }}$ is described in [55,56], and its supersonic approximation and subsonic approximation

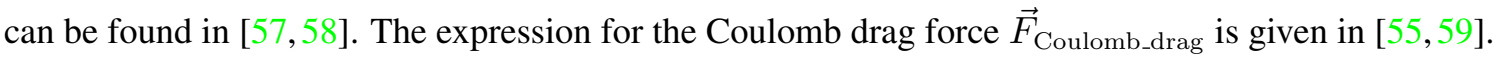

First, we discuss the gravitational environment for dust particles in circumjovian space. Because Jupiter rotates rapidly, it has a shape of an oblate spheroid. As a result, the even zonals of spherical harmonics in the gravity field are the dominant perturbations of the central gravity field. The gravitational perturbations from the four inner moons (Adrastea, Metis, Amalthea and Thebe), four Galilean moons (Io, Europa, Ganymede and Callisto), and the irregular moons are important for dust evolving in their orbital ranges. The solar gravitational perturbation is important for dust grains from the irregular moons.

The Jovian magnetic environment was studied by many researchers, which is needed to calculate the Lorentz force. Important recent models include the O6 model [60], the Ulysses model [61], the VIP4 model [62], the Amalthea model [63], the VIT4 model [64], and the VIPAL model [65]. The Jovian magnetic field is not a pure dipole field. Especially inside the Jovian ring system, the quadrupole and octupole terms are important for the dust dynamics [66,67].

The ambient plasma affects the dust dynamics in terms of dust charging and plasma drag. Many plasma models have been developed [68-72]. Here we discuss the frequently cited global plasma models, the DG1 model [68] and its updated version the DG2 model [72]. The DG1 model [68] was mainly based on the data from the Pioneer and Voyager spacecraft. The DG2 model [72] was updated by data from the Voyager spacecraft and the Galileo spacecraft [69-71]. According to these two models, the Jovian plasma environment is divided into three populations: radiation belt electrons and protons (0.1-100 MeV), warm plasma (0.1-100 keV), and cold electrons, protons and heavy ions (1-100 eV). The cold heavy ion species include $\mathrm{O}^{+}, \mathrm{O}^{++}, \mathrm{S}^{+}, \mathrm{S}^{++}, \mathrm{S}^{+++}$and $\mathrm{Na}^{+}[68,72]$, which are the most important species for plasma drag. In the Jovian plasma environment, the dust particles are charged [73]. The charging currents include electron and ion currents, secondary electron emission and photoelectron emission [73]. For the small stream particles, the dust surface potential is size-dependent [74] because of the small-size effect of the secondary electron emissions. For small grains the charging becomes a stochastic process, dealing with absorption of single unit charges.

For dust particles in the region of the Trojan asteroids, the dominant forces are solar gravity, solar radiation pressure, solar wind drag, Poynting-Robertson drag, the solar wind induced Lorentz force, and gravitational perturbations from Jupiter and other planets. The equation of motion of Trojan dust particles in the heliocentric inertial frame can be expressed as $[43,53,75]$

$$
\begin{aligned}
\ddot{\vec{r}}_{\odot}= & -\frac{G m_{\odot}}{r_{\odot}^{3}} \vec{r}_{\odot}+\sum_{i=1}^{N_{\mathrm{P}}} G M_{\mathrm{P}_{i}}\left(\frac{\vec{r}_{\mathrm{dP}_{i}}}{r_{\mathrm{dP}_{i}}^{3}}-\frac{\vec{r}_{\mathrm{P}_{i}}}{r_{\mathrm{P}_{i}}^{3}}\right)+\frac{Q}{m_{\mathrm{g}}}\left(\dot{\vec{r}}_{\odot}-\vec{v}_{s w}\right) \times \vec{B} \\
& +\frac{3 Q_{\mathrm{S}} Q_{\mathrm{pr}_{\mathrm{r}}} \mathrm{AU}^{2}}{4 r_{\odot}^{2} \rho_{\mathrm{g}} r_{\mathrm{g}} c}\left\{\left[1-(1+s w) \frac{\dot{r}_{\odot}}{c}\right] \hat{\vec{r}}_{\odot}-(1+s w) \frac{\dot{\vec{r}}_{\odot}}{c}\right\} .
\end{aligned}
$$

Here, $\vec{r}_{\odot}$ is the heliocentric position vector of the particle, $N_{\mathrm{P}}$ is the number of the planets that are included as gravitational perturbers, $M_{\mathrm{P}_{i}}$ is the mass of the $i$ th planet, $\vec{r}_{\mathrm{dP}_{i}}$ is the vector pointing from the grain to the $i$ th planet, $\vec{r}_{\mathrm{P}_{i}}$ is the position vector of the $i$ th planet, $\vec{B}_{\odot}$ is the local interplanetary magnetic field, $\vec{v}_{s w}$ is the velocity of the solar wind, and $s w$ defines the ratio of solar wind drag relative to the Poynting-Robertson drag.

The Sun, Jupiter and the Trojan particle form the traditional three-body problem. The solar gravity coupled with Jupiter's gravity tends to make the particles move as their sources in tadpole orbits. The Lorentz force due to the solar wind is induced by the charged particles moving in the interplanetary magnetic field. A description of a model for the interplanetary magnetic field can be found in $[75,76]$. 
Astrodynamics

https://mc03.manuscriptcentral.com/astrod

\section{Dust in the region of the Galilean moons}

Dust particles in the region of the Galilean moons were detected by the DDS onboard the Galileo spacecraft [21,22, 77] (see Figure 3), for which the Galilean moons Io, Europa, Ganymede and Callisto are likely the dominant sources [21, 22]. Dust particles are ejected from the surfaces of these moons by hypervelocity impacts of interplanetary micrometeoroids (see Figure 4 for the illustration of this process). These ejecta form dust clouds in the vicinity of the Galilean moons [16,34,78].

The initial size distribution of the dust ejecta follows a power law

$$
p\left(r_{\mathrm{g}}\right) \propto r_{\mathrm{g}}^{-q} .
$$

The exponent $q$ is the slope of the differential production rate of the dust ejecta. The value $q=3.4$ is consistent with measurements of the dust clouds around the Galilean moons by the Galileo DDS [16,78]. A steeper value $q=3.73$ is indicated by measurements of the dust cloud enshrouding the Earth Moon by the Lunar Dust Experiment (LDEX) instrument onboard the Lunar Atmosphere and Dust Environment Explorer (LADEE) spacecraft [79].

A fraction of the ejected particles escape into circumjovian space, and are distributed in the region of the Galilean moons. Magnetospherically captured interplanetary and interstellar particles also contribute to the dust population in this region [21,35,36]. Besides, by analyzing the Galileo DDS data [80], it was inferred that a fraction of dust particles in this region may come from grains transported inward from Jupiter's outer irregular moons [81], particles transported outward from Jupiter's gossamer rings, as well as focused interplanetary and interstellar dust. Moreover, cometary dust can be captured as it was for comet Shoemaker-Levy 9 [82].

The detected dust number density in the region of the Galilean moons is in the order of $10^{2}-10^{3} \mathrm{~km}^{-3}[18$, 21, 22, 77, 83]. The fate of dust particles from the three Galilean moons Europa, Ganymede and Callisto were investigated in [22]. The dust production rates were estimated, and the orbital behavior of submicron- and micronsized particles was analyzed. It was shown that there exists a tenuous ring in the region of the Galilean moons and simulations were compared to the Galileo DDS data. Particles sized $5 \mu \mathrm{m}$ from the surfaces of the Galilean moons were integrated, leading to a model that gives a reasonable fit to the dust detection by the Pioneer 10 and 11 spacecraft [5]. Later, a semi-analytical model was developed to analyze the dynamics and distribution of charged particles in the region of the Galilean moons [84]. High accuracy simulations for a large number of particles of various sizes were performed in [54], including the gravitational $J_{2}, J_{4}$ and $J_{6}$ terms, Lorentz force, solar radiation pressure, PoyntingRobertson drag, plasma drag, and the gravitational perturbations from the Sun and the four Galilean moons; the particle lifetimes, the asymmetry of the dust configuration in the frame rotating with the Sun, the transport of dust between the Galilean moons and to Jupiter, as well as the distribution of orbital elements of the dust grains were investigated.

In the region of the Galilean moons there exists an appreciable fraction of particles on retrograde orbits detected by the Galileo DDS [21,83]. The sources of these particles are (1) captured interplanetary and interstellar dust through exchange of energy and angular momentum with the Jovian magnetosphere [21, 35, 36], (2) particles transported inward from the Jovian outer irregular retrograde moons [80, 81], (3) particles from comet Shoemaker-Levy 9 [82], and (4) grains from Galilean moons with large inclinations excited by solar radiation pressure and Lorentz force [54].

\section{Possible dust plumes on Europa}

Europa is the major scientific target of the Europa Clipper mission, and will also be investigated by the JUpiter ICy moons Explorer mission. Because of its potential habitability, Europa received much interest by researchers in recent 


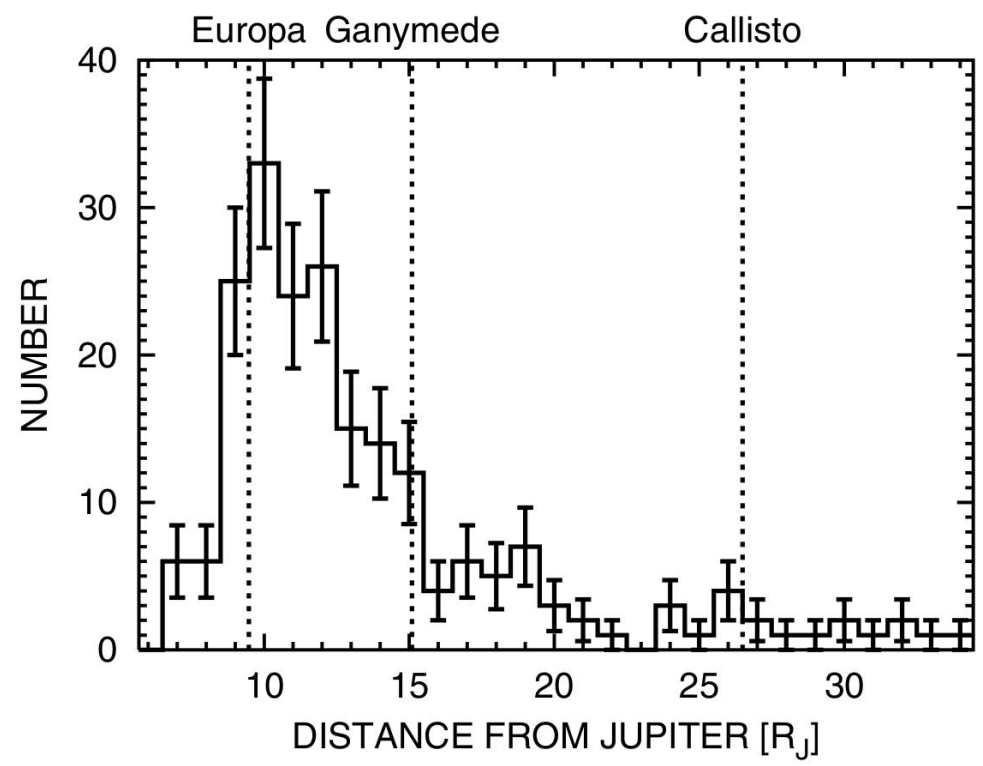

Fig. 3 Number of dust particles detected by the Galileo DDS in the second amplitude range AR2 (from the year 1996 to 2001) vs. the Jovicentric distance. The error bars were overplotted. The dashed lines denote the location of the Galilean moons Europa, Ganymede and Callisto. Reprinted from [22], (c)2002, American Geophysical Union, with permission from John Wiley and Sons.

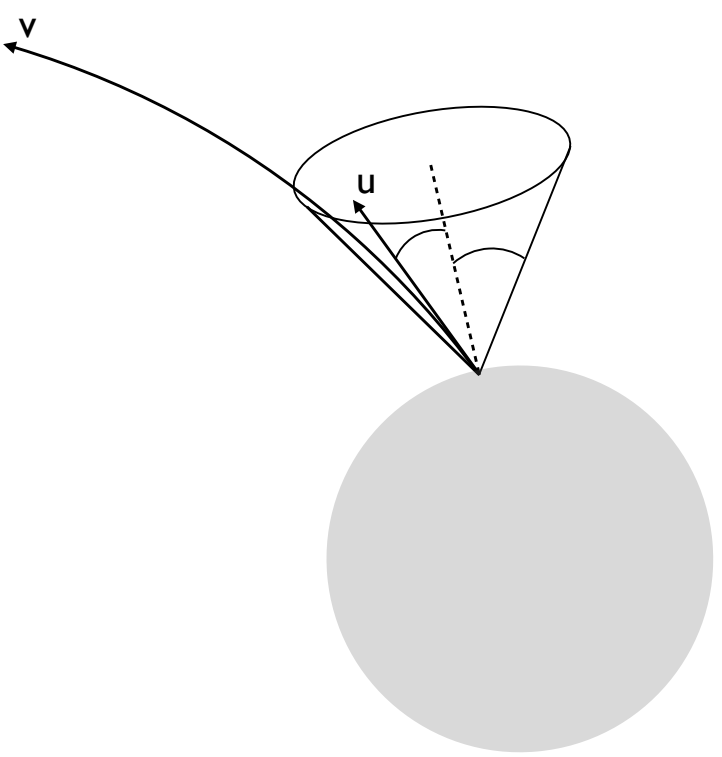

Fig. 4 Schematic diagram of the impactor-ejecta process. A fast interplanetary impactor hits the surface of an atmosphereless body and releases ejecta within a cone from the surface. The symbol $\mathbf{u}$ denotes the initial velocity of a particle at the ejection point on the surface of the source body, and $v$ denotes the velocity of this particle at distance. The arrows indicate the directions of $\mathbf{u}$ and $\mathbf{v}$, respectively. A fraction of the ejecta can escape the gravity of the body and evolve into circumplanetary/circumsolar space, and other ejecta fall back to the body's surface. This mechanism applies to atmosphereless bodies including planetary moons [34,79] and asteroids [85]. 
Astrodynamics

https://mc03.manuscriptcentral.com/astrod

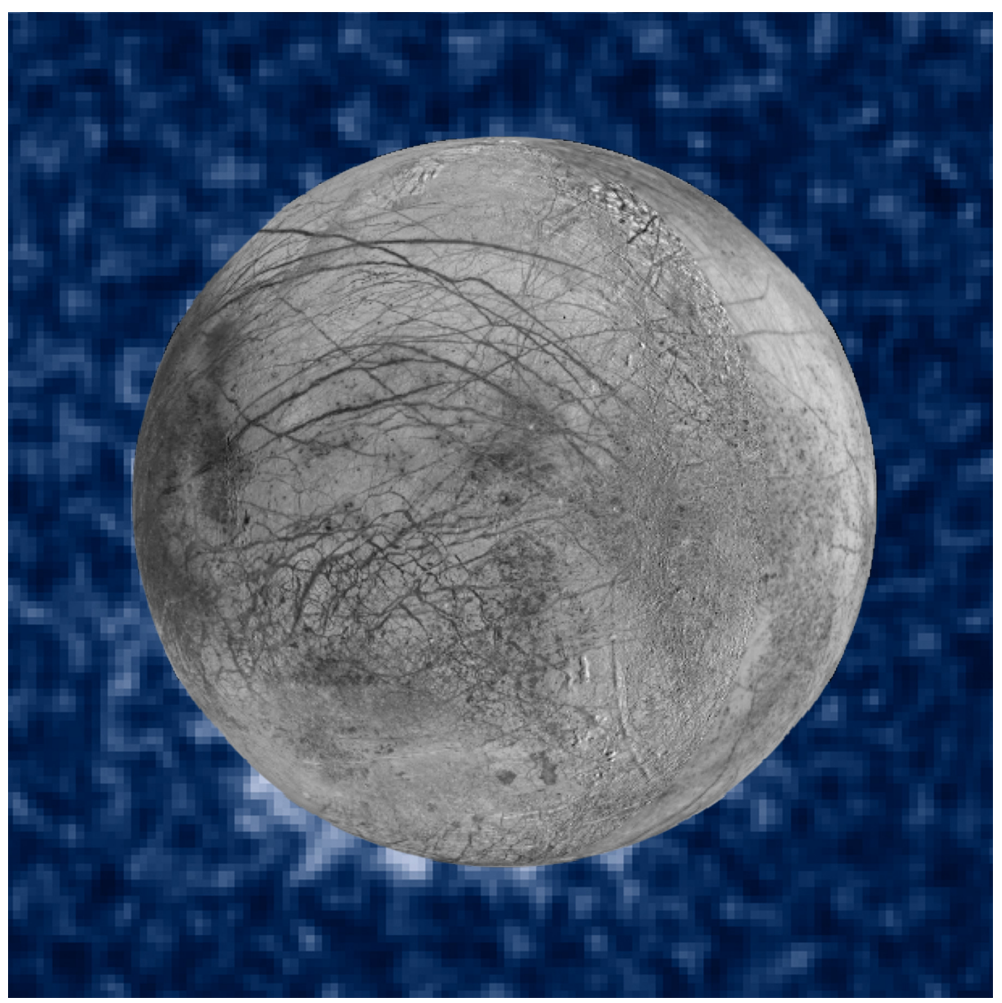

Fig. 5 The suspected Europa's water vapor plume is visible in the lower left of the composite image. The image of the plume was taken by Hubble's Imaging Spectrograph on January 26, 2014. The image of Jupiter's moon Europa was obtained from data returned by Galileo and Voyager. Credit: NASA, ESA, W. Sparks (STScI), and the USGS Astrogeology Science Center.

years, including the plume activity on Europa (Figure 5). In 2012, a water vapor plume was detected in Europa's southern hemisphere for the first time when the satellite was close to orbital apocenter [38]. Follow-up observations showed that orbital location of Europa is not a sufficient condition to detect its plume [39]. Further analysis [86] showed that the orbital location of Europa at true anomaly of about $120^{\circ}$ is a favorable position to detect the plume. Later observations showed that the plume was also detected near Europa's equatorial regions [41].

Europa's plume may contain dust particles [37,40], as it is the case for Saturn's moon Enceladus. The Europa's dust plume was modeled in [40], including Jupiter's gravity with $J_{2}$ term, as well as Europa's gravity. The dust plume structure in terms of the three-dimensional particle number density was derived, and the dust impact rate that can be recorded by the dust instrument onboard spacecraft was calculated [40] (Figure 6). The Europa dust plume was also compared with the Enceladus plume, and the differences were shown. These results are useful to design the SUrface Dust Analyzer instrument onboard the Europa Clipper spacecraft. As it is the case for the Enceladus plume $[87,88]$, the sampling of plume material allows to directly constrain the interior composition of Europa.

\section{Jovian stream particles}

Jovian stream particles are tiny nanometer-sized dust particles that leave the Jovian system at high speed $\left(>200 \mathrm{~km} \mathrm{~s}^{-1}\right)$, and their fluxes are highly variable (see Figure 7; also see Figure 1 in [89] and Figure 8 in [90]). The stream particles were first discovered by the Ulysses spacecraft when approaching Jupiter at a large distance from the planet $[29,91]$, and thus were believed to originate from the Jovian system. The detection of the stream particles also by the 


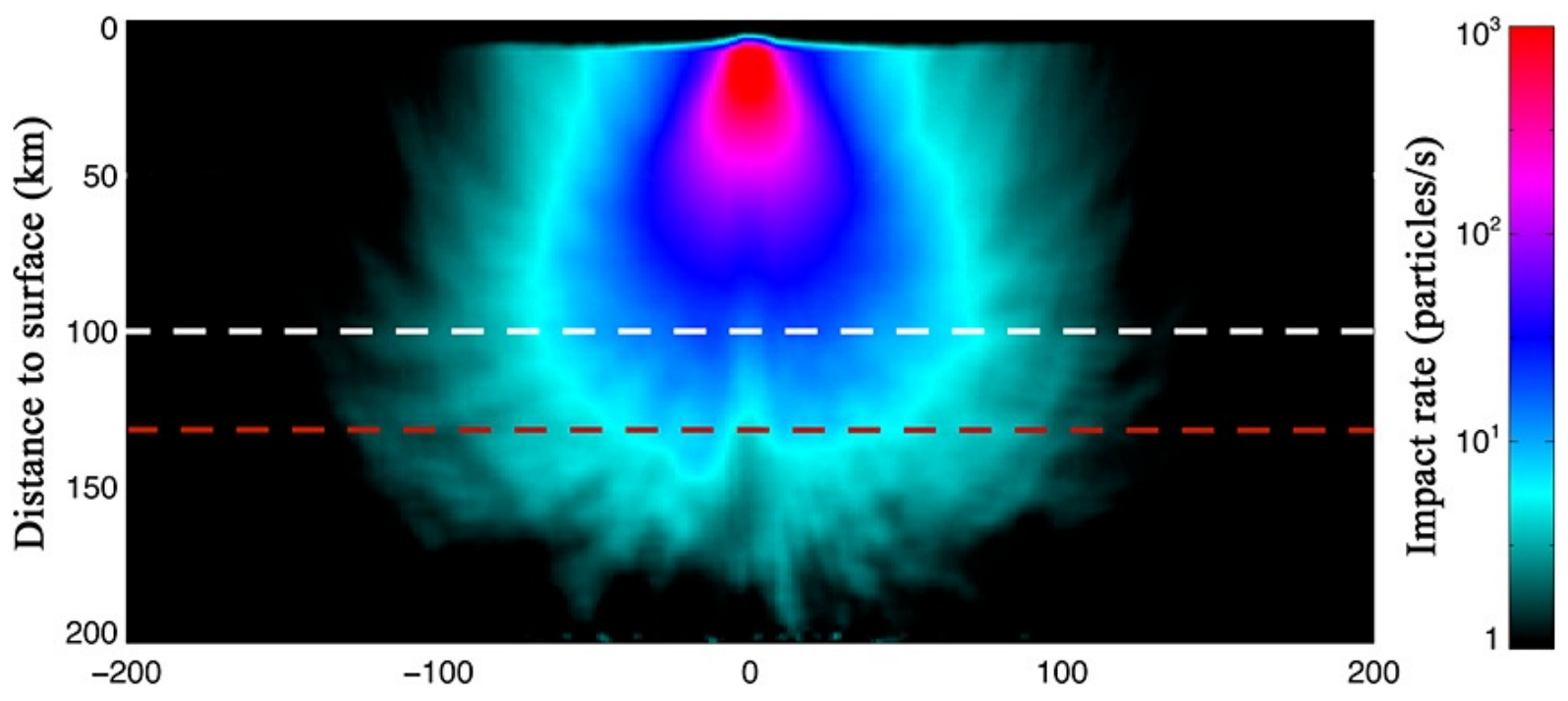

Fig. 6 Vertical impact rate of Europa plume dust that can be recorded by the dust instrument onboard spacecraft. The result was obtained from simulations. The white dashed line denotes the favorable upper altitude of spacecraft, and the red dashed line denotes a parameter-specific larger bound. Reprinted from [40], c)2015, American Geophysical Union, with permission from John Wiley and Sons.

Galileo spacecraft confirmed the Jovian origin [20]. A periodicity in the impact rate of the stream particles recorded by the Galileo DDS was found to compatible with Io's orbital period, which implies that Io is the most likely source for the stream particles [42,92]. This was confirmed by their correlation with Io's volcanic plume activity [89], and by the variation of the stream flux with the Jovian local time [93] (Figure 7), as well as by the compositional analysis of the stream particles performed by the Cosmic Dust Analyzer (CDA) onboard the Cassini spacecraft [94]. This compositional analysis [94] identified sodium chloride $(\mathrm{NaCl})$ as the dominant constituent of the stream particles, with contribution from potassium and sulphur/sulphurous compounds, and possibly a small fraction of silicates or rocky minerals.

The surface equilibrium potential for the Jovian stream particles depends strongly on grain size [74]. Due to their small size and the dependence of the Lorentz force on the inverse square of the grain size, their trajectories are strongly affected by the Lorentz force induced by the Jovian magnetic field [95-98] and by the interplanetary magnetic field $[20,29,91,99]$. The stability of small dust grains in the Jovian magnetic field were analyzed, and the stability boundaries for these small particles were evaluated [100,101]. The charging of the stream particles in Io's cold plasma torus was discussed, where the stream particles are charged negatively by the capture of the cold electrons [102]. In that study, the maximum size of stream particles that can escape from Io was also calculated. The escaping stream particles get recharged positively in the Jovian magnetosphere due to the strong secondary electron current and are eventually ejected from the Jovian system by the Lorentz force [95,96]. The analysis of the Ulysses data showed that the observed pattern for the stream particles is closely correlated with the compression regions in the interplanetary magnetic field (regions of enhanced magnetic field) within a few AU from Jupiter [103].

The stream particles may be a significant source for non-icy material on the Galilean moons. Therefore, their characterization is important to understand the contribution of other mechanisms that bring non-icy material to the surface from the interior of the moons. 


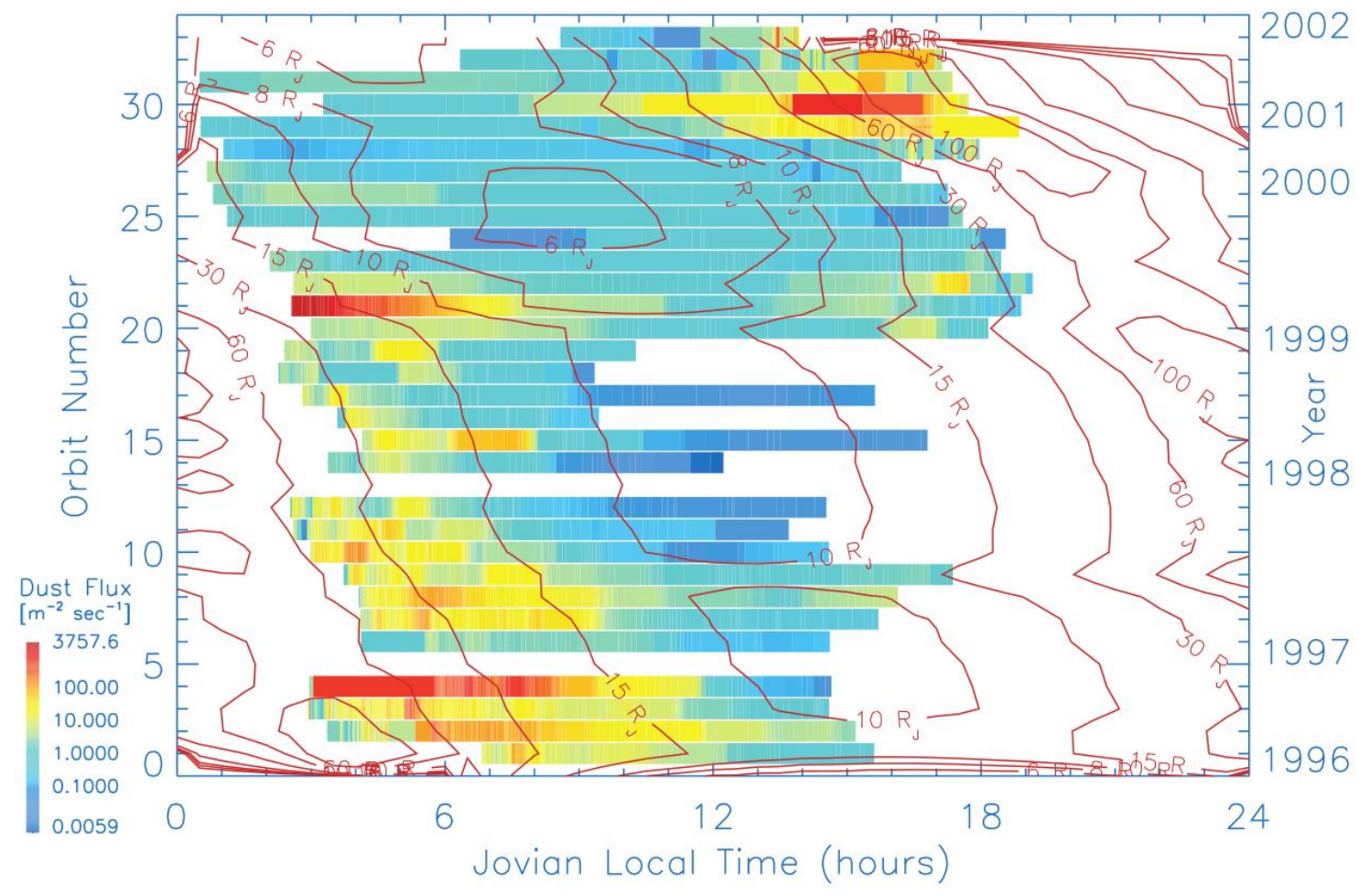

Fig. 7 Measured stream fluxes by the Galileo DDS from the year 1996 to 2002 (along 33 spacecraft orbits around Jupiter) vs. the Jovian local time. The solid line in red denotes the Jovicentric distance $r$. The values of the stream fluxes were scaled by multiplying by a factor of $\left(r / 6 R_{\mathrm{J}}\right)^{2}$. Reprinted from [93], (c)2003, American Geophysical Union, with permission from John Wiley and Sons. 


\section{Dust in the outer region of the Jovian system}

In the outer region of the Jovian system (about $[50,300] R_{\mathrm{J}}$ from Jupiter), there exists a dust population with a nearly constant number density of about $10 \mathrm{~km}^{-3}$, which was detected by the Galileo DDS [23]. The primary source of this dust population is believed to be the Jovian outer irregular moons, which generate dust particles by impacts of interplanetary micrometeoroids. The dominant perturbation forces for particles in this region are solar radiation pressure, Poynting-Robertson drag, solar gravity, and the Lorentz forces induced by the Jovian magnetic field and the interplanetary magnetic field. The dynamics of dust particles in this region was analyzed for submicron- and micronsized particles by including solar radiation pressure, Poynting-Robertson drag and solar gravity [23]. The fate of large debris from the outer, irregular moons was studied in [81]. In their simulations, the motion of particles in the range of $[5,200] \mu \mathrm{m}$ (diameter) influenced by Jupiter's oblateness, solar radiation pressure, Poynting-Robertson drag and planetary perturbations were integrated, and the efficiency and timescale of particle accretion on the Galilean moons Callisto, Ganymede, Europa and Io was analyzed. It was suggested that the dark color of the surfaces of the outer three Galilean moons is primarily due to accumulation of these particles from the outer irregular moons [81]. The accumulation rates could be highest for Callisto, intermediate for Ganymede, and least for Europa, explaining the gradient in coloration of the surfaces of these moons.

\section{Trojan dust}

Trojan asteroids consist of two swarms of asteroids that share the orbit of Jupiter. At the time of this writing, there are more than four thousand Jovian $L_{4}$ Trojan asteroids and more than two thousand $L_{5}$ Trojan asteroids that have been discovered (Jet Propulsion Laboratory Small-Body Database Search Engine (https://ssd.jpl.nasa.gov/ sbdb_query.cgi); IAU Minor Planet Center (https: / /www. minorplanetcenter.net/iau/lists/ JupiterTrojans.html)). To date there was no spacecraft mission to conduct reconnaissance of the Trojan swarms. The Lucy spacecraft (scheduled for launch in 2021) will be the first mission to fly by the Jupiter Trojan asteroids [52].

There exist only few studies of dust particles in the region of Jupiter Trojan asteroids. The non-detection of infrared brightness by the Cosmic Background Explorer (COBE) satellite [104] gives an upper limit of $6 \times 10^{13} \mathrm{~m}^{2}$ for the total cross section of particles roughly larger than $10 \mu \mathrm{m}$ (radius) in the region of the $L_{5}$ Trojans [105]. The dominant dust creation mechanisms are interplanetary micrometeoroid impacts on the surfaces of the Trojan asteroids [43]. In previous research [43], the production rate of the Trojan dust particles was calculated, and a numerical model was developed for the orbital motion of Trojan dust including the forces mentioned above. The evolution of these particles in the size range of $[0.5,32] \mu \mathrm{m}$ (radius) was simulated, and the configuration of Trojan dust in form of arcs was derived [43] (see Figure 8). It was also found that the simulated number density is consistent with the upper limit obtained from the COBE data [104]. Because the dynamics of the Trojan dust is affected by non-gravitational perturbation forces, including solar radiation pressure, solar wind drag, Poynting-Robertson drag and the solar wind Lorentz force, the distribution of the orbital parameters of the Trojan dust differs from that of the Trojan asteroids [106]. Especially, in that study [106], the bimodal pattern of the semi-major axis distribution for Trojan dust was revealed. It was also shown that the distribution peak of the longitude of pericenter for the Trojan asteroids is about 60 degrees larger than Jupiter's longitude of pericenter, while for Trojan dust this difference in the longitude of pericenter is less than 60 degrees. The dynamical behavior of $2 \mu \mathrm{m}$ (diameter) particles trapped in the Jupiter 1:1 resonance was analyzed in [107,108]. A collisional numerical code was used to simulate the evolution of the Trojan dust particles with diameters in the range of $[5,500] \mu \mathrm{m}$, including the effect of the Poynting-Robertson drag [45]. 


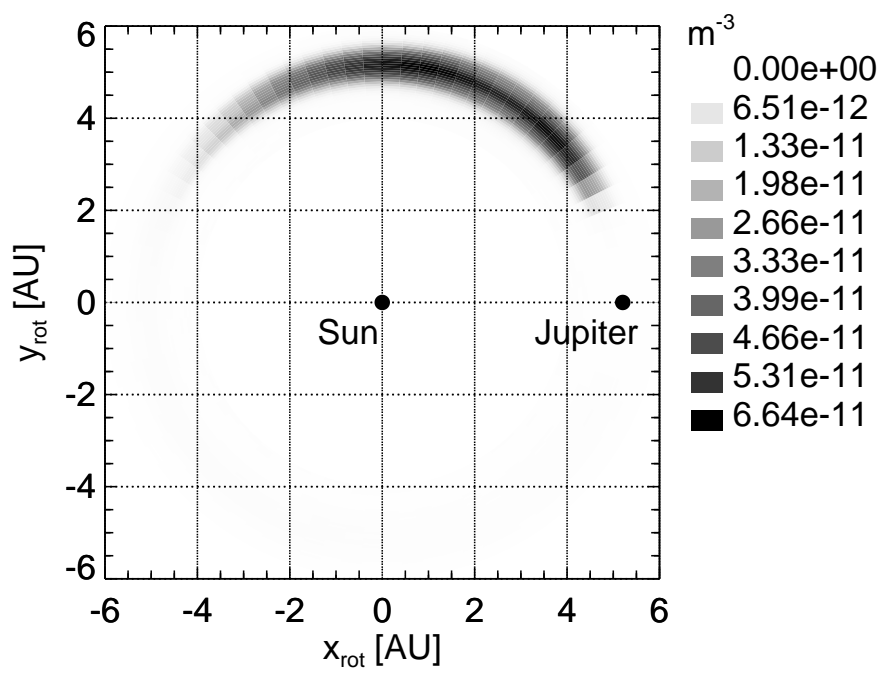

Fig. 8 Number density of dust particles ejected from the $L_{4}$ Trojan asteroids in the frame rotating with Jupiter. The result was calculated from long-term simulations for particles larger than $0.5 \mu \mathrm{m}$, and was averaged vertically over $[-0.55,0.55] \mathrm{AU}$. The Sun lies at the origin, and Jupiter lies at the positive $x$ axis with a heliocentric distance of about 5.2 AU. Credit: Liu and Schmidt, A\&A, 609, A57, 2018 [43], reproduced with permission from Astronomy \& Astrophysics, CESO.

Zimmer and Grogen integrated the orbital evolution of Trojan dust particles between 10 and $90 \mu$ m (diameter) for 500,000 years, and analyzed their fate [44]. They considered the solar gravity, planetary gravitational perturbations, solar radiation pressure, Poynting-Robertson drag and solar wind drag.

\section{Conclusions}

In the 2020s, there are two Jupiter missions and one Trojan mission scheduled for launch: ESA's JUpiter ICy moons Explorer mission, as well as NASA's Europa Clipper mission and Lucy mission to the Trojan asteroids. Especially onboard Europa Clipper, there is a dust instrument, the SUrface Dust Analyzer. An understanding of the Jovian dust environment will be important for the implementation of these missions, and in turn they will inform the preliminary modeling effects. In this review paper, we have summarized the results from previous observations and modeling work for Jovian dust outside the ring system including: particles in the region of the Galilean moons, Europa's dust plume, Jovian stream particles, grains in the outer region of the Jovian system, and dust in the region of the Jupiter Trojans. All relevant perturbation forces, the orbital evolution, and the spatial distributions of dust were discussed.

\section{Acknowledgements}

This work was supported by the European Space Agency under the project "Jovian Micrometeoroid Environment Model" (JMEM) (contract number: 4000107249/12/NL/AF) at the University of Oulu, and by the Academy of Finland under the project "Earth and Near-Space System and Environmental Change". 


\section{References}

[1] Humes D, Alvarez J, O’Neal R, Kinard W. The interplanetary and near-Jupiter meteoroid environments. Journal of Geophysical Research, 1974, 79(25): 3677-3684.

[2] Humes D, Alvarez J, Kinard W, O’neal R. Pioneer 11 meteoroid detection experiment: preliminary results. Science, 1975, 188(4187): 473-474.

[3] Smith BA, Soderblom LA, Johnson TV, Ingersoll AP, Collins SA, Shoemaker EM, Hunt G, Masursky H, Carr MH, Davies ME, et al.. The Jupiter system through the eyes of Voyager 1. Science, 1979, 204(4396): 951-972.

[4] Owen T, Danielson GE, Cook AF, Hansen C, Hall VL, Duxbury TC. Jupiter's rings. Nature, 1979, 281: 442446.

[5] Zeehandelaar DB, Hamilton DP. A local source for the Pioneer 10 and 11 circumjovian dust detections. In H Krüger, A Graps, editors, Proceedings of 'Dust in Planetary Systems', Kauai, Hawaii, USA2007, $103-106$.

[6] Showalter M, Burns JA, Cuzzi J, Pollack JB. Discovery of Jupiter's 'gossamer' ring. Nature (ISSN 0028-0836), 1985, 316: 526-528.

[7] Showalter M, Burns JA, Cuzzi J, Pollack JB. Jupiter's ring system - New results on structure and particle properties. Icarus, 1987, 69: 458-498.

[8] Throop H, Porco C, West R, Burns J, Showalter M, Nicholson P. The jovian rings: new results derived from Cassini, Galileo, Voyager, and Earth-based observations. Icarus, 2004, 172(1): 59-77.

[9] Showalter MR, de Pater I, Verbanac G, Hamilton DP, Burns JA. Properties and dynamics of Jupiter's gossamer rings from Galileo, Voyager, Hubble and Keck images. Icarus, 2008, 195(1): 361-377.

[10] Ockert-Bell ME, Burns JA, Daubar IJ, Thomas PC, Veverka J, Belton M, Klaasen KP. The structure of Jupiter's ring system as revealed by the Galileo imaging experiment. Icarus, 1999, 138(2): 188-213.

[11] Burns JA, Showalter MR, Hamilton DP, Nicholson PD, de Pater I, Ockert-Bell ME, Thomas PC. The formation of Jupiter's faint rings. Science, 1999, 284(5417): 1146-1150.

[12] McMuldroch S, Pilorz SH, Danielson GE, Team NS. Galileo NIMS Near-Infrared Observations of Jupiter's Ring System. Icarus, 2000, 146(1): 1-11.

[13] Brooks SM, Esposito LW, Showalter M, Throop HB. The size distribution of Jupiter's main ring from Galileo imaging and spectroscopy. Icarus, 2004, 170(1): 35-57.

[14] Krüger H, Grün E, Hamilton DP, Baguhl M, Dermott SF, Fechtig H, Gustafson BA, Hanner MS, Horányi M, Kissel J, Lindblad BA, Linkert D, Linkert G, Mann I, McDonnell JAM, Morfill GE, Polanskey C, Riemann R, Schwehm G, Srama R, Zook HA. Three years of Galileo dust data: II. 1993-1995. Planetary and Space Science, 1998, 47(1): 85-106.

[15] Krüger H, Grün E, Graps A, Bindschadler D, Dermott SF, Fechtig H, Gustafson BA, Hamilton DP, Hanner MS, Horányi M, Kissel J, Lindblad BA, Linkert D, Linkert G, Mann I, McDonnell JAM, Morfill GE, Polanskey C, Schwehm G, Srama R, Zook HA. One year of Galileo dust data from the Jovian system: 1996. Planetary and Space Science, 2001, 49(1): 1285-1301. 
[16] Krüger H, Krivov AV, Sremčević M, Grün E. Impact-generated dust clouds surrounding the Galilean moons. Icarus, 2003, 164(1): 170-187.

[17] Krüger H, Bindschadler D, Dermott SF, Graps AL, Grün E, Gustafson BA, Hamilton DP, Hanner MS, Horányi M, Kissel J, Lindblad BA, Linkert D, Linkert G, Mann I, McDonnell JAM, Moiss1 R, Morfill GE, Polanskey C, Schwehm G, Srama R, Zook HA. Galileo dust data from the jovian system: 1997-1999. Planetary and Space Science, 2006, 54(9-10): 879-910.

[18] Krüger H, Hamilton DP, Moissl R, Grün E. Galileo in-situ dust measurements in Jupiter's gossamer rings. Icarus, 2009, 203(1): 198-213.

[19] Krüger H, Bindschadler D, Dermott SF, Graps AL, Grün E, Gustafson BA, Hamilton DP, Hanner MS, Horányi M, Kissel J, Linkert D, Linkert G, Mann I, McDonnell JAM, Moissl R, Morfill GE, Polanskey C, Roy M, Schwehm G, Srama R. Galileo dust data from the jovian system: 2000 to 2003. Planetary and Space Science, 2010, 58(7): 965-993.

[20] Grün E, Baguhl M, Hamilton DP, Riemann R, Zook HA, Dermott S, Fechtig H, Gustafson BA, Hanner MS, Horányi M, et al.. Constraints from Galileo observations on the origin of Jovian dust streams. Nature, 1996, 381: 395-398.

[21] Thiessenhusen K. Dust Grains around Jupiter-The Observations of the Galileo Dust Detector. Icarus, 2000, 144(1): 89-98.

[22] Krivov AV, Krüger H, Grün E, Thiessenhusen KU, Hamilton DP. A tenuous dust ring of Jupiter formed by escaping ejecta from the Galilean satellites. Journal of Geophysical Research, 2002, 107(E1): 2-1.

[23] Krivov AV, Wardinski I, Spahn F, Krüger H, Grün E. Dust on the outskirts of the Jovian system. Icarus, 2002, 157(2): 436-455.

[24] Porco CC, West RA, McEwen A, Del Genio AD, Ingersoll AP, Thomas P, Squyres S, Dones L, Murray CD, Johnson TV, Burns JA, Brahic A, Neukum G, Veverka J, Barbara JM, Denk T, Evans M, Ferrier JJ, Geissler P, HELFENSTEIN P, Roatsch T, Throop H, Tiscareno M, Vasavada AR. Cassini Imaging of Jupiter's Atmosphere, Satellites, and Rings. Science, 2003, 299(5): 1541-1547.

[25] Brown RH, Baines KH, Bellucci G, Bibring JP, Buratti BJ, Capaccioni F, Cerroni P, Clark RN, Coradini A, Cruikshank DP, Drossart P, Formisano V, Jaumann R, Langevin Y, Matson DL, McCord TB, Mennella V, Nelson RM, Nicholson PD, Sicardy B, Sotin C, Amici S, Chamberlain MA, Filacchione G, Hansen G, Hibbitts K, Showalter M. Observations with the Visual and Infrared Mapping Spectrometer (VIMS) during Cassini's flyby of Jupiter. Icarus, 2003, 164(2): 461-470.

[26] Showalter M, Cheng AF, Weaver HA, Stern SA, Spencer JR, Throop H, Birath EM, Rose D, Moore JM. Clump Detections and Limits on Moons in Jupiter's Ring System. Science, 2007, 318(5848): 232-234.

[27] Poppe A, James D, Jacobsmeyer B, Horányi M. First results from the Venetia Burney Student Dust Counter on the New Horizons mission. Geophys. Res. Lett., 2010, 37(1): 11101.

[28] Grün E, Zook HA, Baguhl M, Fechtig H, Hanner MS, Kissel J, Lindblad BA, Linkert D, Linkert G, Mann IB, et al.. Ulysses dust measurements near Jupiter. Science, 1992, 257(5076): 1550-1552. 
[29] Grün E, Zook HA, Baguhl M, Balogh A, Bame SJ, Fechtig H, Forsyth R, Hanner MS, Horányi M, Kissel J, Lindblad BA, Linkert D, Linkert G, Mann I, McDonnell JAM, Morfill GE, Phillips JL, Polanskey C, Schwehm G, Siddique N, Staubach P, Svestka J, Taylor A. Discovery of Jovian dust streams and interstellar grains by the ULYSSES spacecraft. Nature (ISSN 0028-0836), 1993, 362: 428-430.

[30] Krüger H, Graps AL, Hamilton DP, Flandes A, Forsyth R, Horányi M, Grün E. Ulysses jovian latitude scan of high-velocity dust streams originating from the jovian system. Planetary and Space Science, 2006, 54(9): 919-931.

[31] Meier R, Smith BA, Owen TC, Becklin EE, Terrile RJ. Near Infrared Photometry of the Jovian Ring and Adrastea. Icarus, 1999, 141(2): 253-262.

[32] de Pater I, Showalter MR, Burns JA, Nicholson PD, Liu MC, Hamilton DP, Graham JR. Keck infrared observations of Jupiter's ring system near Earth's 1997 ring plane crossing. Icarus, 1999, 138(2): 214-223.

[33] de Pater I, Showalter MR, Macintosh B. Keck observations of the 2002-2003 jovian ring plane crossing. Icarus, 2008, 195(1): 348-360.

[34] Krüger H, Krivov AV, Hamilton DP, Grün E. Detection of an impact-generated dust cloud around Ganymede. Nature, 1999, 399(6736): 558-560.

[35] Colwell JE, Horányi M, Grün E. Capture of interplanetary and interstellar dust by the Jovian magnetosphere. Science, 1998, 280(5360): 88-91.

[36] Colwell JE, Horányi M, Grün E. Jupiter's exogenic dust ring. Journal of Geophysical Research, 1998, 103(E9): 20023-20030.

[37] Quick LC, Barnouin OS, Prockter LM, Patterson G. Constraints on the detection of cryovolcanic plumes on Europa. Planetary and Space Science, 2013, 86: 1-9.

[38] Roth L, Saur J, Retherford KD, Strobel DF, Feldman PD, McGrath MA, Nimmo F. Transient water vapor at Europa's south pole. Science, 2014, 343(6167): 171-174.

[39] Roth L, Retherford KD, Saur J, Strobel DF, Feldman PD, McGrath MA, Nimmo F. Orbital apocenter is not a sufficient condition for HST/STIS detection of Europa's water vapor aurora. Proceedings of the National Academy of Sciences, 2014, 111(48): E5123-E5132.

[40] Southworth B, Kempf S, Schmidt J. Modeling Europa's dust plumes. Geophysical Research Letters, 2015, 42(24).

[41] Sparks W, Hand K, McGrath M, Bergeron E, Cracraft M, Deustua S. Probing for evidence of plumes on Europa with HST/STIS. The Astrophysical Journal, 2016, 829(2): 121.

[42] Graps AL, Grün E, Svedhem H, Krüger H, Horányi M, Heck A, Lammers S. Io as a source of the jovian dust streams. Nature, 2000, 405(6): 48-50.

[43] Liu X, Schmidt J. Dust arcs in the region of Jupiter's Trojan asteroids. Astronomy \& Astrophysics, 2018, 609: A57. 
[44] Zimmer AK, Grogan K. Orbital evolution of dust particles originating from Jupiter's Trojan asteroids. In RS Wilson, R Zanetti, DL Mackison, O Abdelkhalik, editors, AAS/AIAA Spaceflight Mechanics Meeting, volume 152 of Advances in the Astronautical Sciences, Santa Fe, New Mexico, USA2014.

[45] De Elia G, Brunini A. Studying the jovian Trojan dust. Astronomy \& Astrophysics, 2010, 512: A65.

[46] de Pater I, Hamilton D, Showalter M, Throop H, Burns J. The Rings of Jupiter. In MS Tiscareno, CD Murray, editors, Planetary Ring Systems, Cambridge University Press2018.

[47] Burns JA, Simonelli DP, Showalter MR, Hamilton DP, Porco CD, Throop H, Esposito LW. Jupiter's ringmoon system. In F Bagenal, TE Dowling, WB McKinnon, editors, Jupiter: The Planet, Satellites and Magnetosphere, Cambridge University Press2004, 241-262.

[48] Miner ED, Wessen RR, Cuzzi JN. Planetary ring systems, Springer Science \& Business Media2007.

[49] Krüger H, Horányi M, Krivov AV, Graps AL. Jovian dust: streams, clouds and rings. In F Bagenal, TE Dowling, WB McKinnon, editors, Jupiter: The Planet, Satellites and Magnetosphere, Cambridge University Press2004, 219-240.

[50] Plaut J, Barabash S, Bruzzone L, Dougherty M, Erd C, Fletcher L, Gladstone R, Grasset O, Gurvits L, Hartogh P, et al.. Jupiter Icy Moons Explorer (JUICE): Science Objectives, Mission and Instruments. In Lunar and Planetary Science Conference, volume 45, 2014, 2717.

[51] Phillips CB, Pappalardo RT. Europa Clipper mission concept: Exploring Jupiter's ocean moon. Eos, Transactions American Geophysical Union, 2014, 95(20): 165-167.

[52] Levison H, Olkin C, Noll K, Marchi S, Team L. Lucy: Surveying the Diversity of the Trojan Asteroids: The Fossils of Planet Formation. In Lunar and Planetary Science Conference, volume 48, 2017.

[53] Burns JA, Lamy PL, Soter S. Radiation forces on small particles in the solar system. Icarus, 1979, 40(1): $1-48$.

[54] Liu X, Sachse M, Spahn F, Schmidt J. Dynamics and distribution of Jovian dust ejected from the Galilean satellites. Journal of Geophysical Research: Planets, 2016, 121(7): 1141-1173.

[55] Draine BT, Salpeter EE. On the physics of dust grains in hot gas. Astrophys J, 1979, 231: 77-94.

[56] Banaszkiewicz M, Fahr HJ, Scherer K. Evolution of dust particle orbits under the influence of solar wind outflow asymmetries and the formation of the zodiacal dust cloud. Icarus, 1994, 107(2): 358-374.

[57] Morfill GE, Grün E, Johnson TV. Dust in Jupiter's magnetosphere: Physical processes. Planetary and Space Science, 1980, 28(12): 1087-1100.

[58] Dikarev VV. Dynamics of particles in Saturn's E ring: Effects of charge variations and the plasma drag force. Astronomy and Astrophysics, 1999, 346: 1011-1019.

[59] Northrop T, Birmingham T. Plasma drag on a dust grain due to Coulomb collisions. Planetary and space science, 1990, 38(3): 319-326.

[60] Connerney J. Magnetic fields of the outer planets. Journal of Geophysical Research: Planets, 1993, 98(E10): 18659-18679. 
[61] Dougherty MK, Balogh A, Southwood D, Smith EJ. Ulysses assessment of the Jovian planetary field. J. Geophys. Res., 1996, 101(A): 24929-24942.

[62] Connerney JEP, Acuna MH, Ness NF, Satoh T. New models of Jupiter's magnetic field constrained by the Io flux tube footprint. J. Geophys. Res., 1998, 103(A): 11929-11940.

[63] Randall BA. An Improved Magnetic Field Model for Jupiter's Inner Magnetosphere Using a Microsignature of Amalthea. J. Geophys. Res., 1998, 103: 17535.

[64] Connerney JEP. Planetary magnetism. In G Schubert, T Spohn, editors, Treatise on Geophysics, Elsevier2007, 243-280.

[65] Hess SLG, Bonfond B, Zarka P, Grodent D. Model of the Jovian magnetic field topology constrained by the Io auroral emissions. Journal of Geophysical Research, 2011, 116(A5): A05217.

[66] Schaffer L, Burns JA. The dynamics of weakly charged dust: Motion through Jupiter's gravitational and magnetic fields. Journal of Geophysical Research, 1987, 92(A3): 2264-2280.

[67] Burns JA, Schaffer LE, Greenberg RJ, Showalter MR. Lorentz resonances and the structure of the Jovian ring. Nature, 1985, 316: 115-119.

[68] Divine N, Garrett HB. Charged particle distributions in Jupiter's magnetosphere. Journal of Geophysical Research, 1983, 88(A9): 6889-6903.

[69] Sittler E, Strobel DF. Io plasma torus electrons: Voyager 1. Journal of Geophysical Research: Space Physics, 1987, 92(A6): 5741-5762.

[70] Frank L, Paterson W. Galileo observations of electron beams and thermal ions in Jupiter's magnetosphere and their relationship to the auroras. Journal of Geophysical Research: Space Physics, 2002, 107(A12).

[71] Bagenal F, Delamere PA. Flow of mass and energy in the magnetospheres of Jupiter and Saturn. Journal of Geophysical Research: Space Physics, 2011, 116(A5).

[72] Garrett HB, Kim W, Belland B, Evans R. Jovian plasma modeling for mission design. Pasadena, CA: Jet Propulsion Laboratory, National Aeronautics and Space Administration. JPL Publication 15-11. http:// hdl. handle. net/2014/45478, 2015.

[73] Horányi M. Charged dust dynamics in the solar system. Annual Review of Astronomy and Astrophysics, 1996, 34(1): 383-418.

[74] Dzhanoev A, Schmidt J, Liu X, Spahn F. Charging of small grains in a space plasma: Application to Jovian stream particles. Astronomy \& Astrophysics, 2016, 591: A147.

[75] Gustafson BÅS. Physics of zodiacal dust. Annual Review of Earth and Planetary Sciences, 1994, 22: 553-595.

[76] Landgraf M. Modeling the motion and distribution of interstellar dust inside the heliosphere. Journal of Geophysical Research, 2000, 105(A5): 10303-10316.

[77] Krüger H, Grün E. Dust en-route to jupiter and the galilean satellites. In S Green, I Williams, J McDonnell, N McBride, editors, Dust in the Solar System and other Planetary Systems, volume 15 of COSPAR Colloquia Series, Pergamon2002, $144-159$. 
[78] Krüger H, Krivov AV, Grün E. A dust cloud of Ganymede maintained by hypervelocity impacts of interplanetary micrometeoroids. Planetary and Space Science, 2000, 48(15): 1457-1471.

[79] Horányi M, Szalay JR, Kempf S, Schmidt J, Grün E, Srama R, Sternovsky Z. A permanent, asymmetric dust cloud around the Moon. Nature, 2015, 522(7556): 324-326.

[80] Soja RH, Hamilton DP, Altobelli N. A new analysis of Galileo dust data near Jupiter. Planetary and Space Science, 2015, 109: 76-91.

[81] Bottke WF, Vokrouhlickỳ D, Nesvornỳ D, Moore JM. Black rain: The burial of the Galilean satellites in irregular satellite debris. Icarus, 2013, 223(2): 775-795.

[82] Horányi M. New jovian ring? Geophysical Research Letters, 1994, 21(11): 1039-1042.

[83] Soja RH, Altobelli N, Krüger H, Sterken VJ. Dust environment predictions for the ESA L-class mission JUICE. Planetary and Space Science, 2013, 75: 117-128.

[84] Sachse M. A planetary dust ring generated by impact-ejection from the Galilean satellites. Icarus, 2017.

[85] Szalay JR, Horányi M. The Impact Ejecta Environment of Near Earth Asteroids. The Astrophysical Journal Letters, 2016, 830(2): L29.

[86] Rhoden AR, Hurford TA, Roth L, Retherford K. Linking Europa's plume activity to tides, tectonics, and liquid water. Icarus, 2015, 253: 169-178.

[87] Postberg F, Kempf S, Schmidt J, Brilliantov NV, Beinsen A, Abel B, Buck U, Srama R. Sodium salts in E-ring ice grains from an ocean below the surface of Enceladus. Nature, 2009, 459(7): 1098-1101.

[88] Postberg F, Schmidt J, Hillier JK, Kempf S, Srama R. A salt-water reservoir as the source of a compositionally stratified plume on Enceladus. Nature, 2011, 474(7): 620-622.

[89] Krüger H. Jovian dust streams: A monitor of Io's volcanic plume activity. Geophys. Res. Lett., 2003, 30(21): 2101.

[90] Krüger H, Linkert G, Linkert D, Moissl R, Grün E. Galileo long-term dust monitoring in the jovian magnetosphere. Planetary and Space Science, 2005, 53(11): 1109-1120.

[91] Zook HA, Grün E, Baguhl M, Hamilton DP, Linkert G, Liou JC, Forsyth R, Phillips JL. Solar Wind Magnetic Field Bending of Jovian Dust Trajectories. Science, 1996, 274(5): 1501-1503.

[92] Krüger H, Grün E, Graps A, Lammers S. Observations of Electromagnetically Coupled Dust in the Jovian Magnetosphere. Astrophys Space Sci, 1999, 264(1): 247-256.

[93] Krüger H, Horányi M, Grün E. Jovian dust streams: Probes of the Io plasma torus. Geophys. Res. Lett., 2003, 30(2): $30-1$.

[94] Postberg F, Kempf S, Srama R, Green SF, Hillier JK, McBride N, Grün E. Composition of jovian dust stream particles. Icarus, 2006, 183(1): 122-134.

[95] Horányi M, Morfill G, Grün E. Mechanism for the acceleration and ejection of dust grains from Jupiter's magnetosphere. Nature, 1993, 363(6425): 144-146. 
[96] Horányi M, Morfill G, Grün E. The dusty ballerina skirt of Jupiter. Journal of Geophysical Research, 1993, 98(A12): 21245-21251.

[97] Horányi M, Grün E, Heck A. Modeling the Galileo dust measurements at Jupiter. Geophys. Res. Lett., 1997, 24: 2175 .

[98] Grün E, Krüger H, Graps AL, Hamilton DP, Heck A, Linkert G, Zook HA, Dermott SF, Fechtig H, Gustafson BA, Hanner MS, Horányi M, Kissel J, Lindblad BA, Linkert D, Mann I, McDonnell JAM, Morfill GE, Polanskey C, Schwehm G, Srama R. Galileo observes electromagnetically coupled dust in the Jovian magnetosphere. J. Geophys. Res., 1998, 103(E): 20011-20022.

[99] Hamilton DP, Burns JA. Ejection of dust from Jupiter's gossamer ring. Nature, 1993, 364(6439): 695-699.

[100] Jontof-Hutter D, Hamilton DP. The fate of sub-micron circumplanetary dust grains I: Aligned dipolar magnetic fields. Icarus, 2012, 218(1): 420-432.

[101] Jontof-Hutter D, Hamilton DP. The fate of sub-micron circumplanetary dust grains II: Multipolar fields. Icarus, 2012, 220(2): 487-502.

[102] Flandes A. Dust escape from Io. Geophys. Res. Lett., 2004, 31(1): 16802.

[103] Flandes A, Krüger H, Hamilton DP, Valdés-Galicia J, Spilker L, Caballero R. Magnetic field modulated dust streams from Jupiter in interplanetary space. Planetary and Space Science, 2011, 59(13): 1455-1471.

[104] Kuchner MJ, Reach WT, Brown ME. A search for resonant structures in the zodiacal cloud with COBE DIRBE: The Mars wake and Jupiter's Trojan clouds. Icarus, 2000, 145(1): 44-52.

[105] Jewitt DC, Trujillo CA, Luu JX. Population and size distribution of small Jovian trojan asteroids. The Astronomical Journal, 2000, 120(2): 1140.

[106] Liu X, Schmidt J. Comparison of orbital properties of Jupiter Trojan asteroids and Trojan dust. Astronomy \& Astrophysics, 2018, 614: A97.

[107] Liou JC, Zook HA, Jackson A. Radiation pressure, Poynting-Robertson drag, and solar wind drag in the restricted three-body problem. Icarus, 1995, 116(1): 186-201.

[108] Liou JC, Zook HA. An asteroidal dust ring of micron-sized particles trapped in the 1:1 mean motion resonance with Jupiter. Icarus, 1995, 113(2): 403-414. 